\title{
Comparison of the sensitivity and specificity of urine cytology, urinary nuclear matrix protein-22 and multitarget fluorescence in situ hybridization assay in the detection of bladder cancer
}

\author{
ELIJAH O. KEHINDE ${ }^{1}$, FAHD AL-MULLA ${ }^{2}$, KUSUM KAPILA $^{2}$ \& JEHORAM T. ANIM ${ }^{2}$ \\ ${ }^{1}$ Departments of Surgery (Division of Urology), and ${ }^{2}$ Pathology, Faculty of Medicine, Kuwait University, Kuwwait
}

\begin{abstract}
Objective. This study aimed to compare the sensitivity, specificity, positive predictive value (PPV) and negative predictive value (NPV) of urine cytology, BladderChek ${ }^{\circledR}$ nuclear matrix protein-22 (NMP22) and UroVysion ${ }^{\mathrm{TM}}$ fluorescence in situ hybridization (FISH) tests in patients with newly diagnosed bladder cancer, those with recurrent bladder cancer, and those with bladder cancer but in remission during surveillance. Material and methods. Voided urine samples obtained from 178 patients with suspected or known bladder cancer about to undergo diagnostic or surveillance cystoscopy and 25 control subjects without the disease were divided into four and used for urine culture and cytology, NMP22 BladderChek and UroVysion FISH tests. The sensitivity, specificity, PPV and NPV for each test were calculated. Comparison was made between the ability of each test to detect bladder cancer in the three category of patients listed. Results. Of the 178 patients with bladder cancer, 43 were newly diagnosed, 58 had recurrent disease and 77 were in remission. The sensitivity of each test in newly diagnosed patients was: urine cytology 28\%, NMP22 88\% and FISH 80\%; and in patients with recurrent disease: urine cytology 33\%, NMP22 57\% and FISH 85\%. The mean specificity for urine cytology, NMP22 and FISH was $95 \%$, $67 \%$ and $48 \%$, respectively. Conclusion. Of the tests used in the study for detection of bladder cancer, NMP22 appeared to be most costeffective and rapid, with relatively high sensitivity and specificity in all categories of patients. The NMP22 test may be considered a new gold standard for the assessment of patients with known or suspected bladder cancer.
\end{abstract}

Key Words: Bladder cancer, diagnosis, NMP22, urine cytology, UroVysion FISH

\section{Introduction}

For patients diagnosed with bladder cancer close surveillance using urine cytology and cystoscopy is indicated to identify tumour recurrence and prevent disease progression. Cystoscopy and urine cytology are the current standard monitoring tools for superficial bladder cancer. Cytology exhibits variable sensitivity depending on tumour grade, with the lowest sensitivity for low-grade tumours [1]. Interpretation of urine specimens also depends on the skill of the examiner [2]. The sensitivity of cystoscopy is limited to the tumour that can be visualized, and flat in situ carcinomas are often missed. Frequent follow-up cystoscopies are expensive and cause some discomfort for the patients. Consequently, a non-invasive, objective and easy-to-perform diagnostic test that detects bladder tumours and has a high specificity could improve the follow-up of patients with superficial bladder cancer [3-8]. Furthermore, the assessment of patients with haematuria-sensitive urine-based marker assays, particularly those that provide pointof-care results, may not only improve the diagnosis of bladder cancer, but also shorten the time to make a diagnosis [3-5]. Numerous candidate urinary biomarkers for detecting bladder cancer have been developed in the past 10 years. The most intensively studied markers include the BTA Stat Test, nuclear matrix protein-22 (NMP22), UroVysion ${ }^{\mathrm{TM}}$ fluorescence in situ hybridization (FISH) and

Correspondence: E. O. Kehinde, Department of Surgery (Division of Urology), Kuwait University, PO Box 24923,13110 Safat, Kuwait. Tel: +965 25319475. Fax: +965 25319597. E-mail: ekehinde@hotmail.com, E-mail: ekehinde@hsc.edu.kw 
survivin. As there is no consensus among urologists about the best tests to incorporate into routine clinical practice, there is a need to continue to assess the utility of these markers in different parts of the world.

The ideal urinary tumour biomarker should have the following features: high sensitivity, i.e. low false negatives, and high specificity, i.e. low false positives. A tumour marker with low sensitivity will result in underdiagnosis, impaired cancer control and false hope. A tumour marker with low specificity will produce unnecessary anxiety and unwarranted investigations. Urine cytology, introduced into clinical practice by Papanicolaou and Marshall in 1945, has a sensitivity of about $30 \%$ and a specificity of about $85 \%$ in most centres, and is operator dependent [9].

The UroVysion FISH assay (Vysis, IL, USA) is a multitarget assay that detects aneuploidy of chromosomes 3, 4 and 17 and loss of the 9p21 band in exfoliated cells in urine from patients with transitional cell carcinoma (TCC) [3,8]. The NMP22 test detects elevated amounts of nuclear mitotic apparatus protein, a component of the nuclear matrix essential for cell division that is released into urine at cell death $[5,6,8]$. UroVysion FISH and NMP22 BladderChek ${ }^{\circledR}$ test (Matritech, Newton, MA, USA) have been reported to have higher sensitivity and specificity than urine cytology in patients with cystoscopic and histological TCC either at initial diagnosis or on recurrence. They are both approved by the US Food and Drug Administration (FDA) for use as urinary tumour makers.

The aim of this study was to compare the sensitivity and specificity of urine cytology, urinary NMP22 qualitative test (positive or negative) and UroVysion FISH assay in patients with newly diagnosed bladder cancer, recurrent bladder cancer and known TCC of the bladder but without tumour recurrence during surveillance (i.e. in remission) in a Urology Unit in Kuwait, a Middle Eastern country with a bladder cancer spectrum slightly different from that reported in many Western countries.

\section{Material and methods}

Voided urine samples collected before cystoscopy in 178 patients with or without previously diagnosed TCC of the bladder was subjected to urine culture, urine cytology, urinary NMP22 qualitative test and UroVysion FISH assay in a blinded fashion. There were 25 controls. These were patients with no macroscopic or microscopic haematuria undergoing diagnostic cystoscopy. Most of the controls had nonspecific irritative symptoms. Smokers were excluded from the control arm. This was done to avoid introducing confounding factors in the control arm as some of the smokers may have yet undiagnosed upper tract urothelial tumours. About $80 \%$ of patients with TCC in this centre are smokers. All cystoscopies, bladder biopsies, transurethral resection of bladder tumour (TURBT) and examination of patients under anaesthesia to determine tumour stage were carried out by one experienced consultant urological surgeon (EOK). Only white light cystoscopy was used in this study. Patients had a complete work-up including intravenous urography (IVU), bone scan and computed tomographic (CT) scan of the abdomen and pelvis to stage accurately those with bladder cancer. All laboratory analyses indicated below were carried out in a blinded fashion. No bladder wash urinary specimens were used in this study.

All the patients who underwent cystoscopy had a bladder biopsy. Biopsies were taken as follows. In patients found to have bladder tumours at cystocopy, bladder tumour section and normal-looking sections of the bladder were taken; in patients without any tumours found, a four-quadrant random bladder biopsy was taken in addition to the area of the trigone (i.e. a minimum of four biopsies per patient). Thus, bladder tissue was available from all 203 patients studied. The initial diagnosis of bladder TCC was based on history, radiological findings on IVU or CT scan of a filling defect in the bladder, cystoscopy, TURBT specimens or bladder biopsies. Patients with a known ongoing urinary tract infection (UTI) at the time of cystoscopy, urolithiasis, TCC of the renal pelvis or ureter, and patients with squamous cell or adenocarcinoma of the bladder were excluded from the study. The study received local ethics committee approval and the patients signed relevant informed consent forms.

\section{Preparation of urine samples for cytopathological examination}

Four cytospins were prepared from the urine sample using a ThermoShandon cytocentrifuge. If the cell pellet had a bloody appearance, $30 \mathrm{ml}$ of CytoLyt solution was added. The cytospins were fixed in $95 \%$ ethanol overnight and stored at $-20^{\circ} \mathrm{C}$ until staining was carried out. Papanicolaou staining was done on one of the cytospins. Slides were read as follows: malignant cells present, benign cells only, suspicious cells or atypia. Other significant findings such as the presence of inflammatory cells, fungal organisms (e.g. Candida sp.) or parasites (e.g. Schistosomiasis haematobium) were indicated in the final report. All cytopathological analyses were performed by one experienced cytopathologist (KK). 


\section{Tissue histology}

Following cystoscopy, biopsy samples or TURBT specimens obtained were fixed in $4 \%$ formaldehyde solution and sent for histological examination using routine processing into paraffin and standard haematoxylin and eosin (H\&E) staining techniques. Pathological grade and stage were determined. Tumours were graded according to the World Health Organization (WHO) classification, where G1 and G2 were classified as low-grade and G3 as high-grade tumour. All pathological examinations were carried out by one experienced pathologist (JTA). Categorization of patients into newly diagnosed cases, those with recurrent disease or those in remission was based on both clinical history and tissue histology.

\section{Nuclear matrix protein-22 qualitative assay}

The newer point-of-care version of the NMP22 qualitative assay kits (the BladderChek test; Matritech, Newton, MA, USA) was used. Following the kit manufacturer's instructions, four drops of unprocessed urine was added to the sample well and allowed to react for $30 \mathrm{~min}$. A positive test was indicated by the appearance of a line in the $\mathrm{C}$ and $\mathrm{T}$ sections of the well, as shown in Figure 1 (A, B). Some cases, which after cystoscopy and bladder biopsy turned out to have lowgrade TCC, gave an equivocal NMP22 reading (Figure 1C). In these cases the " $T$ " line of the NMP22 well appeared very faint. For the purpose of this study, such cases were regarded as negative. All NMP22 assay results were read by a trained technician.

\section{UroVysion fluorescence in situ hybridization analysis}

Following the standard protocol as per the kit manufacturer's instructions (Abbott, IL, USA), the FISH technique was successfully developed for detecting chromosomal patterns of normal transitional epithelium and those of TCC of the bladder, as shown in Figure 2. After FISH preparation, the images were captured using a fluorescent AxioPlan microscope operated with Metapher software (Metasystems, Germany). Scoring was performed as recommended by UroVysion Proficiency Panel instructions with at least 21-25 cells counted per patient. A positive FISH result was defined as: (i) five transitional cells or greater with a gain of two or more chromosomes 3, 7 or 17 , and/or (ii) 12 cells or more with 9 p21 deletion. All UroVysion FISH analyses were carried out by one experienced molecular pathologist (FA) who was blinded to the diagnosis.

\section{Non- analysable urine samples}

Some urine specimens were not analysable for the following reasons. For urine cytology, there was little or no cellular material after cytospin to enable the cytopathologist to make a definite diagnosis about the absence or presence of malignant cells. This was true for patients with small bladder cancer or those with very tiny recurrences. For the NMP22 test, some results were equivocal, i.e. neither positive nor negative. For the UroVysion FISH test, for unknown reasons distorted chromosomes or no chromosomes were isolated from some urine samples.

These categories of urine sample were excluded from further analysis, based on the premise that a test would be considered positive or negative if enough
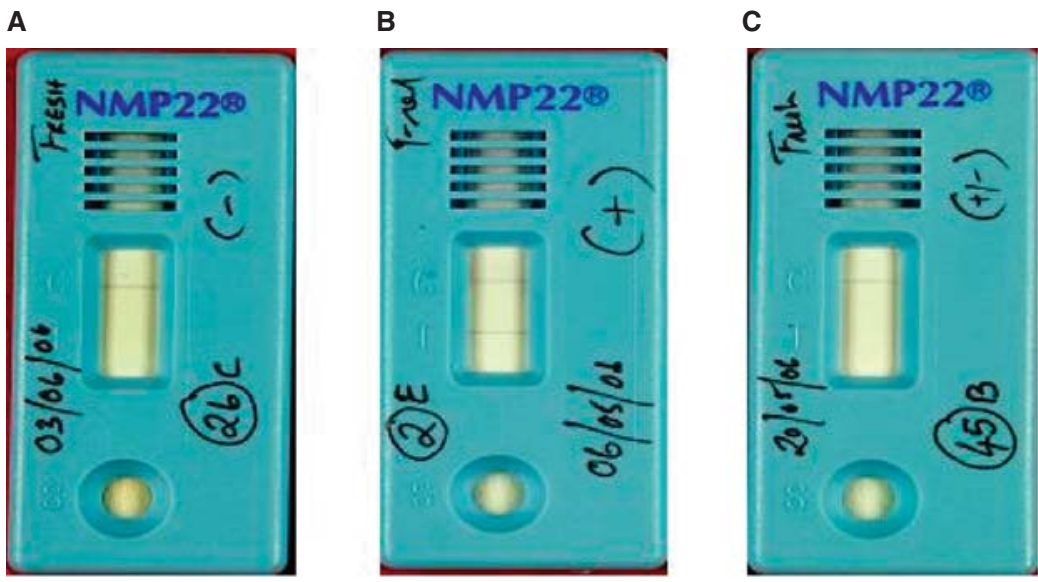

Figure 1. Nuclear matrix protein-22 (NMP22) BladderChek ${ }^{\circledR}$ test. (A) Negative (-); (B) positive (+); (C) equivocal ( \pm ). 

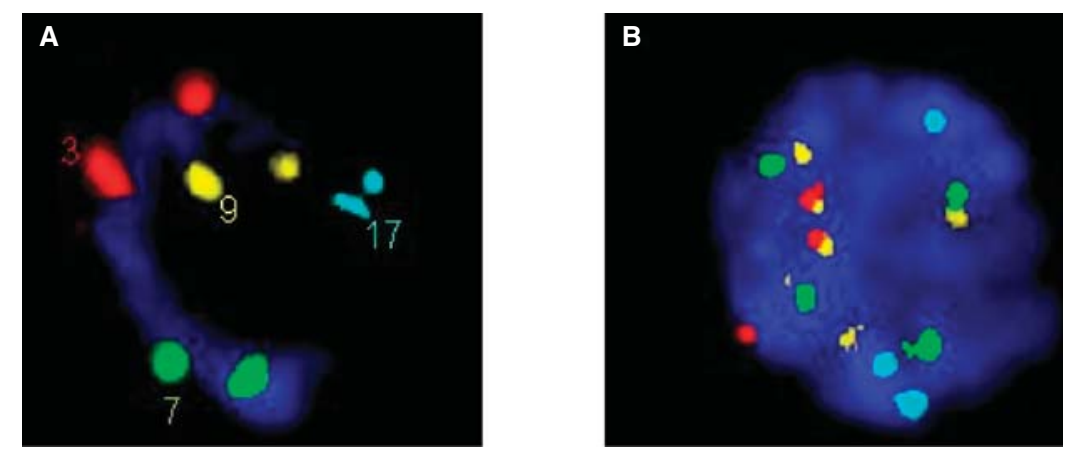

Figure 2. UroVysion test showing fluorescence in situ hybridization (FISH) signals on the interphase nuclei of the bladder epithelial cells in a control subject and a patient with transitional cell carcinoma (TCC) of the bladder. (A) Control: UroVysion FISH test showing two copies of chromosomes 3 (red), 7 (green), 9 (gold) and 17 (aqua) in normal bladder transitional epithelium; (B) positive for TCC of bladder: UroVysion FISH test showing chromosomal aneusomy in chromosomes 3, 7, 9 and 17 with translocation between chromosomes 3 and 9.

cellular or chromosomal material were available to make a correct diagnosis.

\section{Definitions:}

In this study, the following definitions have been used:

- sensitivity $=\mathrm{TP} /(\mathrm{TP}+\mathrm{FN})$ (i.e. tumour positive)

- $\quad$ specificity $=\mathrm{TN} /(\mathrm{TN}+\mathrm{FP})$ (i.e. tumour negative)

- $\mathrm{PPV}=\mathrm{TP} /(\mathrm{TP}+\mathrm{FP})$

- $\mathrm{NPV}=\mathrm{TN} /(\mathrm{TN}+\mathrm{FN})$

- diagnostic accuracy $=(\mathrm{TP}+\mathrm{TN}) / n$, where $n=\mathrm{TP}+\mathrm{FN}+\mathrm{FP}+\mathrm{TN}$

where $\mathrm{TP}=$ true positive, $\mathrm{FN}=$ false negative, $\mathrm{TN}=$ true negative, $\mathrm{FP}=$ false positive, $\mathrm{PPV}=$ positive predictive value, and $\mathrm{NPV}=$ negative predictive value.

The sensitivity, specificity, PPV and NPV of urine cytology, NMP22 and UroVysion FISH assay were compared in patients with newly diagnosed bladder TCC, patients with recurrent bladder TCC and those in remission. Factors affecting the sensitivity of the test (i.e. giving false-positive test results) were analysed. Furthermore, the effect of the final histological grade and stage of tumour on the sensitivity of each urine marker test was analysed.

\section{Statistical analysis}

All data management and analyses were conducted using SPSS software (SPSS, Chicago, IL, USA). The chi-squared $\left(\chi^{2}\right)$ or Fischer's exact test was used to examine the association between categorical variables, and the normal $Z$ test was used to assess the significant difference between two proportions. The $t$ test was used to compare the means of two independent groups. A $p$ value of $<0.05$ was considered statistically significant.

\section{Results}

As shown in Table I, there were 43, 58 and 77 patients with newly diagnosed TCC, recurrent TCC and known TCC but in remission, respectively. Twenty-five patients who underwent cystoscopy but had no bladder outlet obstruction were used as controls. Bladder biopsies in the control group showed neither inflammation nor the presence of urothelial tumour.

Table I shows the mean (range) age, pathological stage and grade of the patients studied. Patients with newly diagnosed bladder TCC had higher stage disease than those who had recurrent disease. Most of the patients presented with low-grade disease (Table I). The patients presented at an earlier age than those reported in the literature. More than $80 \%$ were smokers.

Table II shows the positivity of each test versus the type of patients. The positivity of urine cytology increased from $28 \%$ in patients with newly diagnosed TCC to $33 \%$ in those with recurrent tumour $(p<0.11)$ with a specificity of $95 \%$. The positivity of NMP22 decreased from $82 \%$ for patients with newly diagnosed TCC to $57 \%$ in those with recurrent TCC bladder. NMP22 had an overall specificity of $67 \%$. The ability of FISH to diagnose bladder TCC improved from $81 \%$ in patients with newly diagnosed bladder cancer to $85 \%$ in those with recurrent tumour. The overall specificity of FISH was $48 \%$. In this series of patients, urine cytology had the highest specificity, followed by NMP22 and by UroVysion FISH (Tables I-IV).

Table II also shows the relationship between tumour tissue grade and positivity of each test. The positivity of urine cytology increased with tumour grade, being $70 \%$ for grade 3 and $12 \%$ for grade 1 tumour $(p<0.0001)$. NMP22 and FISH had $100 \%$ positivity regardless of the grade of the tumour. Data in Table II further indicate that when tumour was present, irrespective 
Table I. Pathological grade, stage and profile of patients with bladder cancer.

\begin{tabular}{|c|c|c|c|c|c|c|c|}
\hline & Total no. of cases ${ }^{a}$ & Newly diagnosed & Recurrent & $p^{\mathrm{b}}$ & In remission & Control & Gross total $^{\mathrm{c}}$ \\
\hline \multicolumn{8}{|l|}{ Grade } \\
\hline 1 & 55 & $23(53.5)$ & $32(55.2)$ & 0.09 & $45(58.4)$ & & $100(56.2)$ \\
\hline 2 & 36 & $13(30.2)$ & $23(39.7)$ & 0.06 & $27(35.1)$ & & $63(35.4)$ \\
\hline 3 & 10 & $7(16.3)$ & $3(5.1)$ & 0.001 & $5(6.5)^{\mathrm{d}}$ & & $15(8.4)$ \\
\hline Total $(n)$ & 101 & $43(100)$ & $58(100)$ & & $77(100)$ & 25 & $203(100)$ \\
\hline \multicolumn{8}{|l|}{ Stage } \\
\hline CIS & 6 & $4(9.3)$ & $2(3.4)$ & 0.001 & $9(11.7)$ & & $15(8.4)$ \\
\hline \multicolumn{8}{|l|}{ Superficial } \\
\hline $\mathrm{pT}_{\mathrm{a}}$ & 6 & $4(9.3)$ & $2(3.4)$ & 0.001 & $22(28.6)$ & & $28(15.7)$ \\
\hline $\mathrm{pT}_{1}$ & 54 & $17(39.5)$ & $37(63.9)$ & 0.1 & $42(54.5)$ & & $96(53.9)$ \\
\hline \multicolumn{8}{|l|}{ Invasive } \\
\hline $\mathrm{pT}_{2}$ & 16 & $7(16.3)$ & $9(15.5)$ & 0.1 & $4(5.1)$ & & $20(11.2)$ \\
\hline $\mathrm{pT}_{3}$ & 9 & $5(11.5)$ & $4(6.9)$ & 0.07 & $0(0)$ & & $9(5.1)$ \\
\hline $\mathrm{pT}_{4}$ & 10 & $6(14.0)$ & $4(6.9)$ & 0.001 & $0(0)$ & & $10(5.6)$ \\
\hline Total & 101 & $43(100)$ & $58(100)$ & & $77(100)$ & 25 & $203(100)$ \\
\hline \multicolumn{2}{|c|}{ Age (years), mean (range) } & $53(16-77)$ & $57(23-71)$ & & $59(16-76)$ & $55(20-70)$ & $57(16-76)$ \\
\hline
\end{tabular}

Data are shown as $n(\%)$.

${ }^{a}$ The numbers shown in this column represent the total number of patients recruited and with tissue available for histological analysis following bladder biopsy or transurethral resection of bladder tumour. ${ }^{b}$ Fischer's exact test. ${ }^{c}$ Gross total includes patients with disease and those in remission and control subjects. ${ }^{\mathrm{d}}$ Patients with grade 3 or stage $>\mathrm{p} \mathrm{T}_{1}$ urothelial tumour considered for cystectomy except it is very focal in nature or patient was subjected to partial cystectomy.

of tumour grade, the NMP22 and FISH test had the highest detection rates, while for urine cytology, the higher the tumour grade the higher its sensitivity.

The overall performance characteristics of each test to detect the presence of urothelial tumours are shown in Tables III and IV.

Factors that gave rise to false positive results with the tumour markers were further analysed retrospectively and found to include mild to moderate UTI, presence of urolithiasis, presence of benign prostatic hyperplasia in men and recent treatment with intravesical chemotherapy, particularly bacille CalmetteGuérin (BCG), within 8 weeks of the test.

The mean time taken to obtain results per test in this centre was as follows: urine cytology 44 (range 36-72) h, NMP22 BladderChek test 0.67 (range 0.5$0.83) \mathrm{h}$ and UroVysion FISH 56 (48-72) h. The mean cost per test was about US $\$ 14.00, \$ 24.00$ and $\$ 250.00$ for the NMP22 test, urine cytology and UroVysion FISH, respectively.

Table II. Comparison of positive urine cytology (UC), nuclear matrix protein-22 (NMP22) and fluorescence in situ hybridization (FISH) tests with clinical type and grade of bladder cancer.

\begin{tabular}{llll}
\hline & \multicolumn{1}{c}{ Tests } & \multicolumn{1}{c}{ FISH +ve } & $n=33 / 41(81 \%)$ \\
\cline { 2 - 4 } Clinical type of cancer & \multicolumn{1}{c}{ UC +ve } & $n=31 / 38(82 \%)$ & $n=17 / 20(85 \%)$ \\
Newly diagnosed & $n=10 / 36(28 \%)$ & $n=20 / 35(57 \%)$ & $n=13 / 25(52 \%)$ \\
Recurrent TCC & $n=9 / 27(33 \%)$ & $n=26 / 77(33 \%)$ & \\
In remission (FP) & $n=3 / 56(5.4 \%)$ & $n=25 / 25(100 \%)$ & $n=25 / 25(100 \%)$ \\
Tumour grade & $n=3 / 25(12.0 \%)$ & $n=15 / 15(100 \%)$ & $n=15 / 15(100 \%)$ \\
1 & $n=8 / 15(53.3 \%)$ & $n=10 / 10(100 \%)$ & $n=10 / 10(100 \%)$ \\
\hline
\end{tabular}

Analysis excluded patients found to have too few cells to allow analysis by UC, those with equivocal NMP22 tests and those where no chromosomes were isolated on UroVysion FISH. Most of these were patients with very small tumours or small recurrences. A major cause of false-positive NMP22 and FISH tests was low-grade urinary tract infection $\left(10^{2}-10^{4} \mathrm{cfu} / \mathrm{ml}\right)$ with no organisms cultured. Under tumour grade, comparison was made between the tests in those with definitive positive or negative urine cytology ab initio.

${ }^{\mathrm{a}}$ Gross number $(n)$ of patients recruited per patient category.

TCC $=$ transitional cell cancer; FP $=$ false positive. 
Table III. Performance characteristics of urine cytology (UC), nuclear matrix protein-22 (NMP22) and fluorescence in situ hybridization (FISH) tests in bladder cancer patients in remission.

\begin{tabular}{lccr}
\hline & \multicolumn{3}{c}{ Remission patients (tissue diagnosis) } \\
\cline { 2 - 4 } & UC $(n=56)$ & NMP22 $(n=77)$ & FISH $(n=25)$ \\
\hline False positive & $3(5.4)$ & $26(33)$ & $13(52)$ \\
True negative & $53(95)$ & $51(67)$ & $12(48)$ \\
\hline
\end{tabular}

Data are shown as $n(\%)$.

$n=$ number of tests performed for each tumour marker out of 77 patients in remission. Patients whose urine samples were not used for UC or FISH had very few cells in the urine, in the category of patients with TCC of the bladder in remission.

\section{Discussion}

The ideal bladder cancer tumour marker should be objective, non-invasive, reproducible, and easy and inexpensive to administer and interpret, and must also possess high sensitivity and specificity [6,7]. In addition, it should be possible to assay the marker in voided urine samples. The availability of point-of-care tests resulting in immediate results is highly desirable, since tests that require special processing and analysis, such as enzyme-linked immunosorbent assay (ELISA), reverse-transcription-polymerase chain reaction (RT-PCR) or FISH will lead to delays in the results [7]. A test that permits quantitative assay is also desirable as the changes in the concentration of the marker in the urine may be helpful in clinical decision making, as is the case of prostatespecific antigen (PSA) in patients with prostate cancer. A recent comprehensive literature review by van Rhijn et al. [10] calculated sensitivities and specificities for 17 urine-based bladder tumour markers in addition to urine cytology for patients undergoing surveillance owing to a prior history of urothelial carcinoma. As expected, the sensitivity of all urine markers was higher than that of urine cytology for the detection of recurrent bladder cancer. However, the sensitivity was lower in the detection of recurrence than in the detection of new tumours. The decreased sensitivity for surveillance may be related to decreased tumour stage, grade and size on surveillance compared with screening, as has been described in other reports $[11,12]$. The present findings confirm these previous findings, as shown in Tables I and II. In this study, the performance characteristics of urine cytology, Matritech's NMP22 BladderChek test and UroVysion FISH test were chosen for assessment because the last two are among the few tests currently approved by the US FDA as urinary tumour markers [13]. Furthermore, these two markers are different in terms of cost, ease of use and their mechanisms of action.

The nuclear matrix protein is responsible for cell separation and chromatid regulation during mitosis and is released during apoptotic cell death. The available tests include a qualitative point-of-care test that costs $\$ 10-30$ per test and a quantitative ELISA that costs $\$ 125-150$ per test $[7,14,15]$. NMP22 testing appears to have decreased sensitivity

Table IV. Performance characteristics of urine cytology (UC), nuclear matrix protein-22 (NMP22) and fluorescence in situ hybridization (FISH) tests in patients with bladder cancer.

\begin{tabular}{|c|c|c|c|c|c|c|}
\hline \multirow[b]{5}{*}{ Performance } & \multicolumn{6}{|c|}{ Test } \\
\hline & \multicolumn{2}{|c|}{ UC } & \multicolumn{2}{|c|}{ NMP22 } & \multicolumn{2}{|c|}{ FISH } \\
\hline & \multicolumn{6}{|c|}{ Type of patient } \\
\hline & ND TCC & RCT TCC & ND TCC & RCT TCC & ND TCC & RCT TCC \\
\hline & $\begin{array}{c}(N=43, \\
n=10 / 36)\end{array}$ & $\begin{array}{l}(N=58 \\
n=9 / 27)\end{array}$ & $\begin{array}{c}(N=43, \\
n=31 / 38)\end{array}$ & $\begin{array}{c}(N=35, \\
n=20 / 35)\end{array}$ & $\begin{array}{c}(N=43, \\
n=33 / 41)\end{array}$ & $\begin{array}{c}(N=20, \\
n=17 / 20)\end{array}$ \\
\hline Sensitivity (\%) & 28 & 33 & 82 & 57 & 80 & 85 \\
\hline Specificity (\%) & 95 & 95 & 66 & 67 & 48 & 48 \\
\hline PPV (\%) & 84.9 & 86.8 & 71.3 & 63 & 61 & 62 \\
\hline NPV (\%) & 84.1 & 58.6 & 78.8 & 60.9 & 71.2 & 76 \\
\hline Diagnostic accuracy (\%) & 62 & 64 & 74.5 & 62 & 64.5 & 61.5 \\
\hline
\end{tabular}

$\mathrm{ND}=$ newly diagnosed; RCT $=$ recurrent; $\mathrm{TCC}=$ transitional cell carcinoma; $N=$ gross number of patients recruited in each category; $n=$ number of patients actually analysable in each category; PPV = positive predictive value; NPV = negative predictive value.

Reasons for difference between the tests: UC: small cellular material retrieved after centrifugation, equivocal NMP22 tests and no chromosomes isolated by UroVysion FISH. Most of these were patients with very small tumours or small recurrences. 
in detecting recurrent tumours $(65 \%$ in primary tumours and $45 \%$ in recurrent tumours) [12]. These figures compare favourably with the present finding of NMP22 sensitivity of $82 \%$ and $57 \%$ in patients with newly diagnosed TCC bladder and those with recurrent bladder cancer, respectively. The lower sensitivity with recurrent cancer is presumably due to the lower tumour burden in patients with recurrence and hence a lower quantity of NMP22 in the urine. To improve the sensitivity without sacrificing the specificity of NMP22 in patients with recurrent TCC bladder, perhaps kits that can detect NMP22 values as low as $2 \mathrm{U} / \mathrm{ml}$ can be produced, since the currently available kits are designed to test positive if NMP22 in urine is greater than $6 \mathrm{U} / \mathrm{ml}$ of NMP22. Patients who were categorized as having an equivocal NMP22 result (Figure 1C) were probably those with small low-grade or low-volume recurrences. In addition, as highlighted above, false-positive rates can be as high as $25 \%$ in the presence of non-tumour urological disorders or within weeks of intravesical chemotherapy. A false-positive rate of 33\% was obtained. However, median sensitivity and specificity in surveillance have been reported to be as high as 71 and $73 \%$, respectively [9]. A recent multicentre study assessed the ability of NMP22 to detect recurrent cancer in 668 patients [16]. Recurrent cancer was identified in 103 patients $(15.4 \%)$ and was mostly superficial $(87 \%)$ and low grade (63\%). The combination of NMP22 and cystoscopy has been found to be more sensitive than cystoscopy alone in detecting recurrences (99 vs $91 \%, p=0.005)[9,16]$. NMP22 has a high NPV, which was as high as $91.2 \%$ in some series [17]. In the present study, an NPV of $78.8 \%$ and $60.9 \%$ was found in patients with newly diagnosed TCC and recurrent TCC, respectively. It has higher sensitivity than cytology, with lower specificity (not statistically significant) (Table IV). Therefore, the authors agree with others that it may potentially be used in lieu of cytology in surveillance, offering cost benefits, improved sensitivity and potentially equivalent specificity (in the absence of other genitourinary conditions) [15]. Although a positive result could result in unnecessary cystoscopy and/or biopsy, a negative result may allow lengthening of the interval between surveillance cystoscopies or prescreening which patients require cystoscopy [7].

The FISH UroVysion is a laboratory-based assay that uses colour-specific fluorescent probes against the centromeres on chromosomes 3, 7, 17 and 9 p21 to detect common chromosomal aberrations in exfoliated bladder cancer cells [8]. The test costs approximately \$475-700 per evaluation [14]. FISH has been reported to have a median sensitivity of $79 \%$ and a median specificity of $70 \%$ for surveillance of recurrent tumours [9]. These data compare favourably with the present data in patients undergoing surveillance for TCC bladder, in which a sensitivity of $85 \%$ and a specificity of $48 \%$, respectively, were found. However, FISH has been reported not to perform well for high-grade disease and carcinoma in situ, but sensitivity may not be much better than cytology in the detection of low-grade and lowstage recurrent tumours (36-60\%) [18]. Therefore, its utility in replacing cystoscopy has not yet been clearly established and at best it may be better than cytology in surveillance as it can be less equivocal; however, it is far more expensive. Limitations of the FISH test include its cost, the requirement for intact urothelial cells and trained personnel with sophisticated equipment, and an unstandardized definition of a positive result [7]. Several patients were excluded from analysis in this study because of a lack of sufficient cellular material, particularly in those with recurrent diseases or in remission. This might have affected some of the calculations. The authors agree with Agarwal et al. [7] that, currently, FISH testing should be reserved for select clinical situations rather than used indiscriminately in surveillance.

In this comparative analysis UroVysion FISH had high sensitivity in patients with newly diagnosed $(100 \%)$ or recurrent bladder TCC $(100 \%)$. It had a low specificity of $48 \%$ in all patients with TCC of bladder. Presumably this is due to the fact that most of the patients with TCC of the bladder have some degree of instability of their urothelial chromosomal pattern, giving rise to a high false-positive UroVysion test. This is the subject of further investigations in this centre. NMP22 had higher sensitivity and specificity than cytology in all categories of patients with TCC. NMP22 had lower sensitivity than FISH in patients with newly diagnosed TCC or those with recurrence ( $p$ not significant).

From the data presented and those of others, it can be argued that the current rigorous surveillance strategies in patients with bladder cancer may be imposing excessive diagnostic burden on patients with lowgrade disease for the sake of adequately monitoring those with high-grade disease. This increases the overall cost of management of patients with bladder cancer. Although NMP22 has a high false-positive rate, it is superior to cytology in sensitivity, and with careful patient selection its specificity can be improved. Given its high NPV, it may best be reserved for determining patients in whom it may be possible to extend the interval between subsequent cystoscopies. The critical issue that remains to be answered is whether these markers can reliably replace cystoscopy and prolong the interval between direct visualization without missing a recurrent tumour that could 
potentially progress during the interval. In highrisk patients, one option would be to use a cheap, easy-to-perform marker with high sensitivity, such as NMP22, in all patients undergoing surveillance as an adjunct to cystoscopy. But instead of performing cystoscopy for all patients with a positive NMP22, a more specific marker such as FISH or cytology could be used to clarify the results. A composite picture may then emerge, which would avoid subjecting patients with low-risk disease to unnecessary testing. More prospective studies are needed to prove that low-risk patients can safely have cystoscopies at longer intervals, with or without marker assays replacing cystoscopy, and without affecting their diseasespecific survival. Such a strategy may lead to reduced cost. However, it would have to be tailored to the individual patient, such that the cost savings do not come at the expense of the well-being of patients [6].

Other options worth exploring include the development of NMP22 kits that can detect levels of NMP22 as low as $2 \mathrm{U} / \mathrm{ml}$, which may increase the sensitivity NMP22 without sacrificing its specificity, especially in those with suspected recurrent disease with small tumour burden. An alternative option may be to explore serial measurements of urinary NMP22 concentration and determine its role in detecting recurrence, much like serum PSA in patients with prostate cancer. Finally, combinations of NMP22 and other tumour markers have been shown to increase the sensitivity and the NPV of NMP22 in the detection of recurrence of superficial urothelial tumours $[19,20]$. This stepwise approach of tumour marker determination may be useful in the future to reduce the frequency of surveillance cystoscopies at a reasonable risk, particularly in patients with low-grade superficial tumours [19].

This study has some limitations. Only white light cystoscopy was performed. Narrow-band imaging cystoscopy is known to improve the detection of recurrent superficial bladder cancer compared with white light cystoscopy [21]. In newly diagnosed patients with bladder TCC, the performance characteristic of urinary tumour markers could be improved if analysis was performed in patients at high risk of bladder cancer, such as older patients with gross haematuria and smokers [22]. The exclusion of these factors in the present study might have led to lower specificity and sensitivity of the assessed tumour markers. That is, the specificity of these tests is different depending on the setting in which they are used. For example, the NMP22 test has a much higher specificity when used in the initial assessment of patients with haematuria [22]. This study was carried out in Kuwait, a Middle Eastern country where the spectrum of bladder cancer presentation is different from that in many Western countries. For example, the age range of these patients is much lower than in the West and the majority of patients were smokers. These factors might have affected the results although, as the data showed, not to any significant extent. Therefore, these tumour markers may have a role in the management of patients with urothelial tumours not only in Kuwait, but also in other parts of the world.

In conclusion, at present, it does not appear that the NMP22 BladderChek or the UroVysion FISH test can replace cystoscopy in the initial diagnosis or follow-up of patients with bladder cancer. However, the NMP22 test could replace routine cytology in the initial assessment of patients with suspected urothelial cancer. Urine cytology has higher specificity than NMP22 and FISH in bladder cancer patients without tumour recurrence. FISH may not be an ideal urinary tumour marker for surveillance in patients with bladder cancer because of its low specificity. Overall, NMP22 appears to have the best performance characteristics. Coupled with low cost and quick results, NMP22 may be an ideal tumour marker to replace or complement urine cytology in the management of all categories of patients with urothelial cancer.

\section{Acknowledgements}

This work was supported by Kuwait University research grant MS 02/03. The following technical staff assisted with this project: Mrs Vinsu Jerin and Ms Sarah Jeena John assisted with the reading of NMP22 BladderChek test and Mrs Sindhu Jacob carried out the UroVysion FISH assay under the supervision of F.A. Dr Anjum Memon of the Department of Primary Care, University of Sussex, UK, provided the statistical review. This work also used some resources (mostly the FISH software program) at the Research Core Facility of the Health Sciences Center of Kuwait University, Kuwait (grant nos GM01/01 and GM01/05).

Declaration of interest: The authors report no conflicts of interest. The authors alone are responsible for the content and writing of the paper.

\section{References}

[1] Murphy W. Current status of urinary cytology in evaluation of bladder neoplasms. Hum Pathol 1990;21:886-96.

[2] Raitanen MP, Aine R, Rintala E, Kallio J, Rajala P, Juusela H, et al. FinnBladder Group. Differences between local and review urinary cytology in diagnosis of bladder cancer. An interobserver multicenter analysis. Eur Urol 2002;41:284-9. 
[3] Sarosdy MF, Kahn PR, Ziffer MD, Love WR, Barkin J, Abara EO, et al. Use of a multitarget fluorescence in situ hybridization assay to diagnose bladder cancer in patients with haematuria. J Urol 2006;176:44-7.

[4] Schmitz-Drager BJ, Beiche B, Tirsar LA, Schmitz-Drager C, Bismarck E, Ebert T. Immunocytology in the assessment of patients with asymptomatic microhaematuria. Eur Urol 2007;51:1582-8.

[5] Bott S, Chanawani M, Mostafid H. The use of the NMP22 bladdercheck test for bladder cancer to optimize investigations in a one-stop haematuria clinic. Br J Med Sci Surg Urol 2008;1:126-30.

[6] McNeil BK, Getzenberg RH. Urine-based markers in bladder cancer: future prospects BJU Int 2008;101:668-9.

[7] Agarwal PK, Black PC, Kamat AM. Considerations on the use of diagnostic markers in management of patients with bladder cancer. World J Urol 2008;26:39-44.

[8] Lokeshwar VB, Habuchi T, Grossman HB, Murphy WM, Hautmann SH, Hemstreet GP III, et al. Bladder tumor markers beyond cytology: international consensus panel on bladder tumor markers. Urology 2005;66:35-63.

[9] Papanicolaou GN, Marshall VF. Urine sediment smears as a diagnostic procedure in cancers of the urinary tract. Science 1945;101:519-20.

[10] van Rhijn BW, van der Poel HG, van der Kwast TH. Urine markers for bladder cancer surveillance: a systemic review. Eur Urol 2005;47:736-48.

[11] Miyanaga N, Akaza $\mathrm{H}$, Tsukamoto $\mathrm{S}$, Shimazui $\mathrm{T}$, Ohtani $M$, Ishikawa $S$, et al. Usefulness of urinary NMP22 to detect tumor recurrence of superficial bladder cancer after transurethral resection. Int J Clin Oncol 2003;8: 369-73.

[12] Boman H, Hedelin H, Holmang S. Four bladder tumor markers have a disappointingly low sensitivity for small size and low grade recurrence. J Urol 2002;167:80-3.
[13] Nielsen ME, Schaffer EM, Veltri RW, Schoenberg MP, Getzenberg RH. Urinary markers in the detection of bladder cancer: what's new? Curr Opin Urol 2006;16: $350-5$.

[14] Konety BR. Molecular markers in bladder cancer: a critical appraisal. Urol Oncol 2006;24:326-37.

[15] Sharma S, Zippe CD, Pandrangi L, Nelson D, Agarwal A. Exclusion criteria enhance the specificity and positive predictive value of NMP22 and BTA stat. J Urol 1999;162: 53-7e.

[16] Grossman HB, Soloway M, Messing E, Katz G, Stein B, Kassabian V, Shen Y. Surveillance for recurrent bladder cancer using a point-of-care proteomic assay. JAMA 2006; 295:299-305.

[17] Witjes JA, van der Poel HG, van Balken MR, Debruyne FMJ, Schalken JA. Urinary NMP22 and karyometry in the diagnosis and follow-up of patients with superficial bladder cancer. Eur Urol 1998;33:387-91.

[18] Lokeshwar VB, Selzer MG. Urinary bladder tumor markers. Urol Oncol 2006;24:528-37.

[19] Horstmann M, Patschan O, Hennenlotter J, Senger E, Feil G, Stenzl A. Combinations of urine based tumour markers in bladder cancer surveillance. Scand J Urol Nephrol 2009;43:461-6.

[20] Villicana P, Whiting B, Goodison S, Rosser CJ. Urine based assays for the detection of bladder cancer. Biomark Med 2009;3:265-70.

[21] Herr HW, Donat SM. A comparison of white-light cystoscopy and narrow-band imaging cystoscopy to detect bladder tumour recurrences. BJU Int 2008;102: 1111-14.

[22] Lotan Y, Shariat SF, NMP22 Study Group. Impact of risk factors on the performance of the nuclear matrix protein 22 point-of-care test for bladder cancer detection. BJU Int 2008;101:1362-7. 